\title{
Psychoneuroimmunological aspects of cardiovascular diseases: a preliminary report
}

\author{
DAGMARA BARTCZAK, ŁUKASZ SZYMAŃSKI, PAWEŁ BODERA, WANDA STANKIEWICZ
}

Military Institute of Hygiene and Epidemiology, Warsaw, Poland

\begin{abstract}
Aim of the study: Due to their prevalence and negative social effects, cardiovascular diseases belong to a group of civilization diseases. Previous research suggests comorbidity of heart diseases, mood disorders and impaired cognitive functioning. The aim of this study was to evaluate the psychoneuroimmunological aspects of functioning in patients diagnosed with cardiovascular diseases.

Material and methods: Ten persons, mean age 48.2 years old, diagnosed with primary hypertension, were studied. All of them were treated with beta blockers and ACE inhibitors with unsuccessful therapeutic effect. This group also included 4 subjects with heart rate disturbances. The control group included 10 clinically healthy volunteers in mean age 46.8. All participants had 24-hour ECG monitoring with Holter method in order to evaluate the autonomic activity with time and frequency domain analysis (heart rate variability). Patients also underwent neuropsychological assessment of quality of life and personality traits (EQ-5D, NEO-PI-R, PSS10, SWLS, MHLC). Quantitative evaluation of immune system parameters included: TCD3, TCD4, CD8, CD16/CD56, CD19, HLA-DR+.

Results: The cardiovascular disease group showed significantly lower time and frequency domain parameters $(p<0.05)$ except low/high frequency $(L F / H F)$ power ratio. The heart rhythm disorder group demonstrated significant relationships such as: Quality of life with Total Power of HRV and day-time $L F / H F$ ratio, pNN50 and rMSSD - negative correlation.

Conclusions: 1. In cardiovascular disease patients, activity of the autonomic nervous system is significantly reduced. 2. Impaired modulation of the autonomic nervous system activity affects mood and decreases quality of life. 3. In patients with heart rhythm disturbances, increased sympathetic nervous system activity affects prolonged tension and the immune response.
\end{abstract}

Key words: cardiovascular diseases, HRV, psychoneuroimmunology.

(Cent Eur J Immunol 2016; 41 (2): 209-216)

\section{Introduction}

Due to their prevalence and negative social and economic effects cardiovascular diseases belong to a group of civilization diseases [1]. In the pathogenesis of primary hypertension (HT) and heart rate disturbances (HRD), clinicians stress the impact not only of the increased autonomic nervous system (ANS), immune system and endocrine and paracrine signaling activity but also their close functional and anatomical links [2]. Previous research suggests comorbidity of heart diseases, mood disorders and impaired cognitive functioning [3].

The essence of hypertension is permanent or periodic high blood pressure without an obvious cause. Since 2005, the new definition has been applied, which specifies hypertension as a group of cardio-vascular symptoms, which are the succession of complex and interacting factors. Clinicians emphasize the link between the hypertension and the risk of stroke, coronary heart disease, heart failure, kidney failure and changes in blood vessels. In addition, people with hyperten- sion are more likely to develop cognitive impairments than their peers with blood pressure in the normal range [4]. Controlling the blood pressure is a complex physiological activity dependent on and closely related to the principle of feedback loops, interoperability: renin-angiotensin-aldosterone system (RAA), the sympathetic nervous system, natriuretic peptides, substances produced by the vascular endothelium and the immune system [5].

Arrhythmias, both supraventricular and ventricular, are common and are accompanied by virtually all heart diseases. Additionally, they deteriorate the comfort of life and are the cause of more frequent hospitalizations, morbidity and sometimes sudden death [6]. Whatever the mechanisms directly responsible for the occurrence of heart rate disturbances (abnormal automaticity, triggered activity and re-entry), in induction of arrhythmia, the impact of the increased activation of neurohumoral cascade, including sympathetic activation, the hypothalamic-pituitary-adrenal axis (HPA) and the RAA system is accentuated [7].

Correspondence: Dagmara Bartczak, Military Institute of Hygiene and Epidemiology, Kozielskiego 4, 01-163 Warsaw, Poland,

e-mail: dagmara.bartczak@gmail.com

Submitted: 4.11.2015, Accepted: 16.02.2016 
With the dramatic increase in human life expectancy over the past century, the research of the immune system became crucial. The central role of the thymus as the organ emerging immune competence of the immune system (T cells) has been proved by classic Miller's experience from the early 1960s [8-10]. Hormonal nature of the thymus as the central organ of the immune system is proved not only with intra- but also outside impact on T cells' differentiation process and maturation and also typical for the endocrine system, synergistic and antagonistic relationship with humoral products of the neurohormonal system [11]. Being the recipient of neurohormonal influence, thymus also has opposite affection. Its well-defined hormonal products support the production of hypothalamus trigger hormones. Thymosin $\alpha 1$ stimulates the steroid cascade of the hypothalamic-pituitary-adrenal axis (HPA) and thymosin $\beta 4$ coordinates proper functioning of the hypothalamic-pituitary-gonadal axis [12]. Researchers assume that thymus also affects proper functioning of the hypothalamic-pituitary-thyroid axis [10]. Thymus is an important center of functional links between the immune, endocrine and the nervous system, forming a homeostatic immune-neuro-endocrine network $[13,14]$. These links allow to adjust the immune system activity to the changing needs of the organism at different levels of its development, biological activity and aging in constant confrontation with changing environmental demands. Deficiencies in the thymus activity lead to the disintegration of the neuro-immuno-hormonal network with the onset of immune deficiency symptoms [15].

The aging process causes changes in the immune system. Since cardiovascular diseases are also age related there is an obvious question about the link between them. Recent human and animal studies proved that $\mathrm{T}$ cells play a critical role in cardiovascular diseases. Novel studies showed that in humans as well as in animal models progression of atherosclerosis and coronary artery disease is associated with circulating T cells [8]. What is more, it is known that T cells play a crucial role in the immune response during atherogenesis [9]. Guzik et al. have shown the pathogenic role of $\mathrm{T}$ cells in hypertension. They documented that transfer of $\mathrm{T}$ cells restores hypertension in B cell-lacking RAG1-/- mice, which was previously protected from hypertension [13].

Patients with hypertension have an increased frequency of CD8+CD28- or CD8+CD57+ T cell when compared to healthy controls. Moreover, hypertensive patients have an increased number of CD8 $\mathrm{T}$ cells producing IFN- $\gamma$, TNF- $\alpha$, perforin, or granzyme B in peripheral blood. Based on this research, hypothesis has been suggested, according to which antigenic stimulation of $\mathrm{T}$ cells eventually leads to the loss of CD28 and gain of CD57, and T cells accumulate. The amplified and accumulated cells might sustain the hypertensive response by releasing IFN- $\gamma$ and TNF- $\alpha$ and other proinflammatory cytokines, as well as cytotoxic mediators such as granzymes and perforin [14]. However, it is not yet fully known if T cells are only by- standers or the amplifiers and sustainers of hypertension. For this reason, it is possible that there is a correlation between immune system activity and hypertension and cardiac arrhythmia.

\section{Quality of life}

In recent years, quality of life assessment has become more popular due to its impact on chronic diseases. In medical approach, the term "quality of life" is a parameter that is required in order to study the effects of the implemented treatment of the disease. According to the modern World Health Organization (WHO) definition, "quality of life" is a subjective perception of own circumstances by the patient, according to own objectives, requirements, anxieties, interests and patterns with regard to the system of values and culture [16]. Psychological concepts of quality and satisfaction with life emphasize the importance of not only physical fitness, but also the lack of limitations in effective daily and social activities. Subjective quality of life assessment is determined by the type of the disease, implemented treatment and sometimes by a long diagnostic and therapeutic process [17]. In numerous studies, quality of life of patients suffering from arrhythmia was observed as worse than in healthy individuals [18]. The causes of reduced quality of life in this group of patients are complex. In those untreated, symptoms are directly related to pressure values, or the sense of cardiac arrhythmias such as: cognitive impairments, motor and sensory dysfunctions, sexual problems and sleep disorders, which adversely affect the subjective perception of health [19]. It should be mentioned that multicenter clinical studies focused on the quality of life assessment in particular medical conditions, taking into account the direct effect on the human organism of the primary disease and implemented pharmacotherapy (rarely). The aim of own study was to evaluate the psychoneuroimmunological aspects of functioning in patients diagnosed with cardiovascular diseases. Analysis of obtained results enables evaluation of not only the impact of disease symptoms, but also a proposal of applied effective therapy in the context of psychoneuroimmunological parameter changes.

\section{Material and methods}

Studied groups included 10 patients in mean age 48.2 with primary hypertension. Additionally, in 4 patients concomitant heart rate disturbances have been diagnosed. Inclusion criteria: patients were treated with beta blockers and angiotensin-converting-enzyme inhibitor (ACE) with unsuccessful therapeutic effect; none of the participants used antidepressants or sedative agents; in the studied group, chronic diseases affecting the autonomic nervous system were excluded; all patients had preserved cardiovascular capacity (NYHA functional class I), confirmed with natriuretic peptide proBNP NT-vestibular testing. 
During the diagnostic process all patients underwent complex cardiological diagnostics (including evaluation of severity of HF) and received optimal pharmacotherapy. Additionally, patients also underwent psychological assessment, which included questionnaires in order to assess quality of life and personality traits (EQ-5D, NEO-PI-R, PSS10, SWLS, MHLC). All tests were conducted in an almost identical manner in order to create standard conditions for all patients. Patients had 24-hour ECG monitoring with Holter method in order to evaluate the autonomic activity with time and frequency analysis. Blood samples $(5 \mathrm{ml})$ were used for quantitative immunoassay: TCD3, TCD4, CD8, CD16/CD56, CD19, HLA-DR+.

Parameters of autonomic nervous system activity:

- SDNN - standard deviation of normal-to-normal (NN) intervals,

- rMSSD - the root mean square differences of successive $\mathrm{NN}$ intervals,

- pNN50 - proportion of successive NN intervals greater than $50 \mathrm{~ms}$,

- LF $\left(\mathrm{ms}^{2}\right)$ - low frequency spectral power,

- HF $\left(\mathrm{ms}^{2}\right)$ - high frequency spectral power,

- LF (d); HF (d) - low; high frequency day spectral power,

- LF (n); HF (n) - low; high frequency night spectral power,

- $\mathrm{LF} / \mathrm{HF}$ - the ratio of LF power to HF power,

- TP - total spectral power.

\section{Psychological test characteristics}

\section{$E Q-5 D$}

The EQ-5D questionnaire is a frequently used to assess general quality of life, comprises five dimensions of health (with three answer alternatives each): mobility, ability to self care, ability to undertake usual activities, pain and discomfort, and anxiety and depression. EQ-5D contains two parts - the first includes health opinion in accordance with one of the mentioned criteria, whereas the other - general health opinion.

\section{NEO-PI-R: Revised NEO Personality Inventory}

The NEO-PI-R is a measure of five major domains of personality (Extraversion, Agreeableness, Conscientiousness, Neuroticism, and Openness to Experience) and six facets that define each domain. Taken together, the five domain scales and thirty facet scales of the NEO-PI-R facilitate a comprehensive and detailed assessment of a normal adult personality. The NEO-PI-R is recognized internationally as a gold standard for personality assessment.

\section{PSS10: Perceived stress scale 10}

PSS10 is used to assess perception of stress. Statements are classified on a five-point Likert-type scale from 0 (never) to 4 (very often) and refer to the last month. The higher the total score, the intensity of perceived stress is greater.

\section{SWLS: Satisfaction with Life Scale}

Satisfaction with Life Scale is designed for individual and group study of healthy and sick adults. The scale contains five statements. The test evaluates the degree to which each of them refers to his life in the past. The result of the measurement is the overall sense of satisfaction with life. Average time: 2 minutes.

\section{MHLC: Multidimensional Health Locus of Control Scale}

MHLC is a questionnaire containing 18 statements concerning general expectations in three dimensions of health locus of control: perceptions reflected a belief in the patients' own, powerful others (influences of other people) and coincidence.

Spearman Rank and Pearson Test were used in order to find correlation coefficients as well as significance testing.

The study protocol was approved by the Military Institute of Aviation Medicine (WIML) Ethics Committee with decision number 04/2015.

\section{Results}

The cardiovascular disease group showed a significantly lower time and frequency domain parameters. The spectral components changes of HRV in a healthy person, hypertensive patient and patient with hypertension and heart rhythm disturbances is shown in Figs. 1-3.

In the cardiovascular diseases group, HRV parameters were observed as significantly lower. In the HT and HRD group significantly lower SDANN, LF, LFd (LF value with day activity component) parameters were observed. Autonomic nervous system activity in other time and frequency analysis parameters did not show significant differences.

Differences in the mean values of immune parameters and the results of psychological tests in the studied group, depending on the presence of HRD, are shown in Figs. 4,5 . The percentage of antigen occurrence in the studied group is shown in Table 1. Correlations between immune parameters and parameters of autonomic nervous system activity are shown in Table 2.

Identification of CD4 antigen on $\mathrm{T}$ cell subpopulation and the ratio of CD4/CD8: More relevant than determination of the CD4 and CD8 value is determination of the ratio of CD4 to CD8. The increased ratio of CD4/CD8 is to some extent a reflection of the proportion of Th1-type immune response to $\mathrm{Th} 2$.

The increased ratio of CD4/CD8 is observed in patients with autoimmune diseases.

Identification of CD19 antigen on B lymphocyte subsets: CD19-cells are responsible for production of antibodies of all classes (IgG, $\operatorname{IgM}$ and $\operatorname{IgA}$ ).

Identification of CD16 + CD56 + antigen on natural killer cell subsets: Both natural killer cell CD56+ CD16+ 


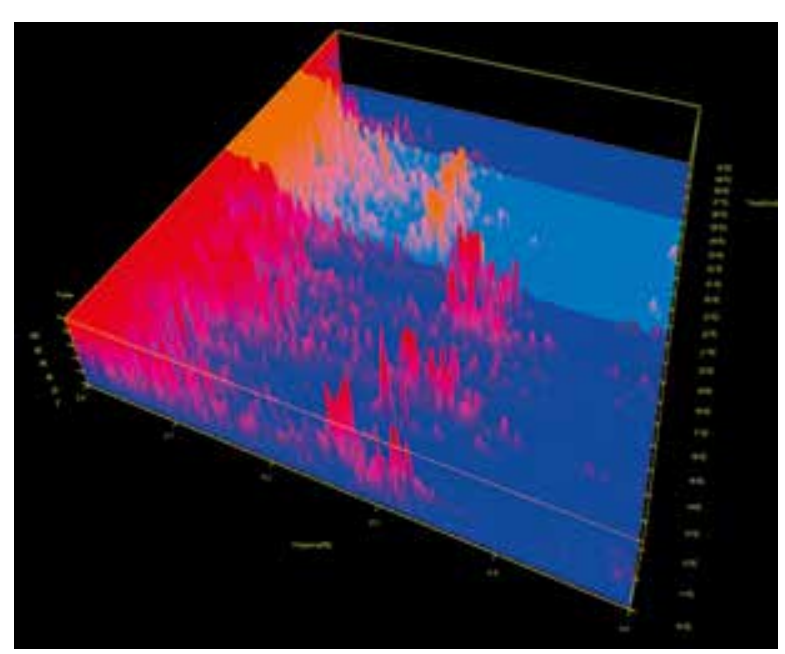

Fig. 1. The spectral component power of HRV in a healthy person

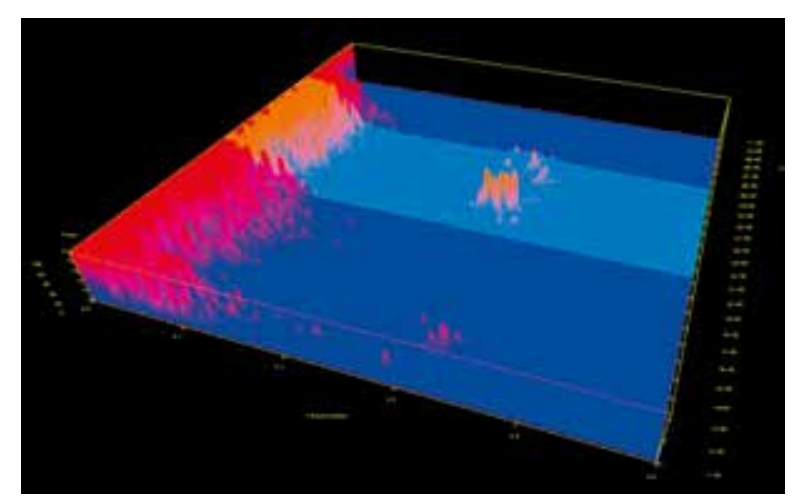

Fig. 3. The spectral component power of HRV in an HT and HRD person

and CD56 + subpopulations are mainly TNF producers. NK cells - CD56+ (CD16-) are capable of significantly higher production of TNF- $\alpha$ than CD56+ CD16+ NK cells which in turn can easily migrate to the tissue.

Mean values of psychological tests are shown in Fig. 5, whereas the correlation between quality of life assessment and HRV parameters are shown in Table 3.

\section{Discussion}

Since Claude Bernard's first lecture in 1865 , a physiological connection between the heart and the brain has been widely studied. Although well-documented species differences, mammals studies have proved that both, primates and humans exhibit direct and indirect pathways linking the frontal cortex with the autonomic nervous system associated with sympathoexcitatory and parasympathoinhibitory effects on the heart [2]. Parasympathetic activity decreases the heart rate and increases heart rate

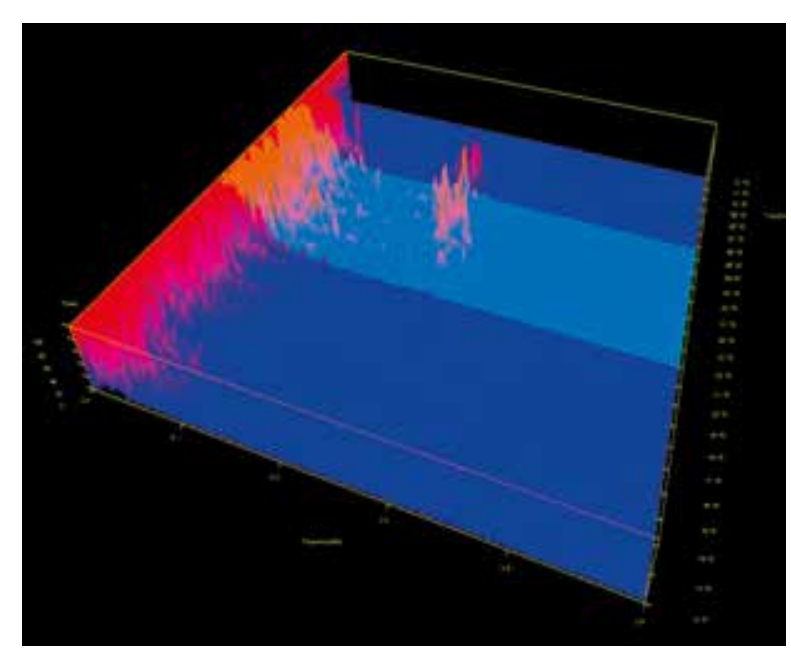

Fig. 2. The spectral component power of HRV in an HT person

variability (HRV) whereas sympathetic activity increases the heart rate and decreases HRV. Neuroanatomical studies displayed inhibitory activity of GABAergic pathways from the prefrontal cortex to the amygdala as well as inhibitory pathways between amygdala and the sympathetic and parasympathetic branches of the autonomic nervous system that modulate heart rate and thus heart rate variability [2]. Other studies using pharmacological and neuroimaging approaches also proved that activity of the prefrontal cortex is associated with vagally mediated HRV [20]. Other clinical studies have proved that dominance of sympathetic nervous system activity is the main cause of major cardiac pathologies observed in neurological catastrophes [21]. On the other hand, researchers indicate that all risk factors, known to favor cardiovascular diseases, are associated with decreased heart rate variability.

Researchers emphasize the influence of individual differences in HRV in physiological, emotional, and cognitive functioning [20]. Neuropsychological studies report a relation between HRV and cognitive regulation - especially, inhibition (inhibit prepotent but inappropriate responses) is crucial for health and effective functioning in enriched, complex environment. Effective cognitive functioning in daily activities involve working memory, sustained attention, inhibition of inappropriate responses, planning [22]. According to the neuroimaging studies, those aspects of behavior (executive functions) are associated with increased blood flow in prefrontal cortex, whereas cognitive deficits might be a result of either aging or negative affective states, or reduced HRV value [23]. Patients often report that subjective cognitive deficits affect health-related quality of life. Cognitive functioning impairment results as decision-making disabilities and thereby limiting effective performance of self-care. In most studies, investigators are using global screening questionnaires to assess cognitive 


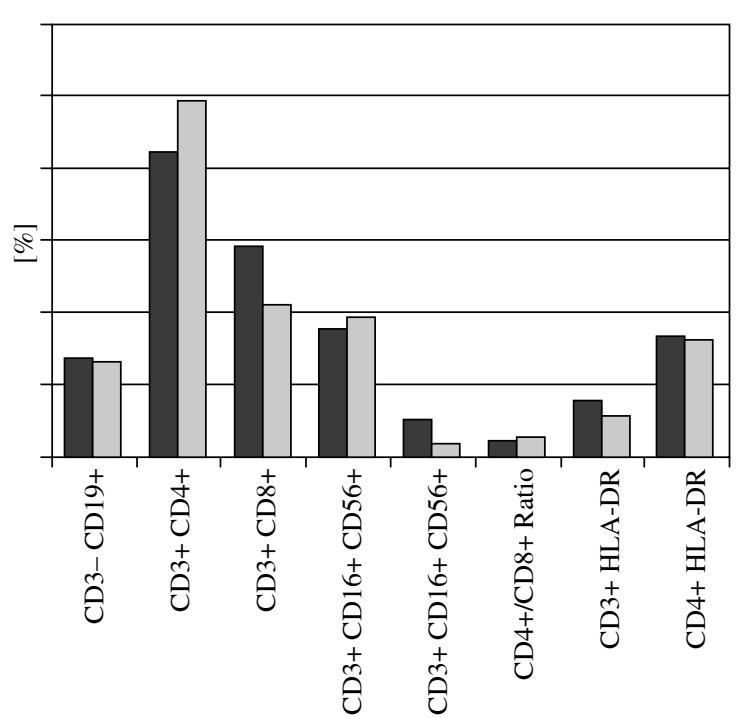

口 HRD 0

$\square$ HRD 1

Fig. 4. Mean values of immune parameters in the studied group

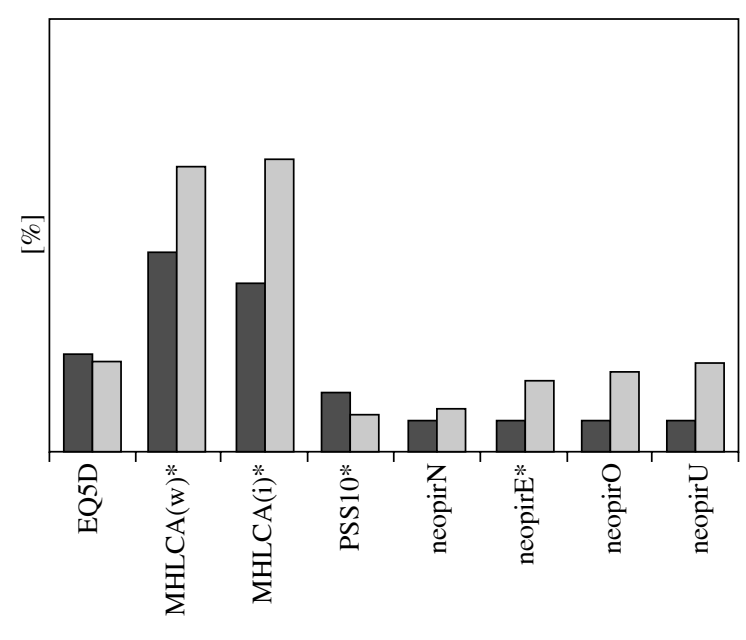

$\square$ HRD 0

$\square$ HRD 1

Fig. 5. Mean values of psychological tests in the studied group

Table 1. Percentage of antigen occurrence in the tested population

\begin{tabular}{|c|c|c|}
\hline Antigen & Tested cell population & Reference value \\
\hline CD3 & T-lymphocyte subpopulation & $64-74 \%$ \\
\hline CD4 & T helper lymphocyte subpopulation & $41-49 \%$ \\
\hline CD8 & cytotoxic T lymphocyte subpopulation & $31-38 \%$ \\
\hline CD4/CD8 & Th1/Th2 ratio & $0.6-2.8 \%$ \\
\hline CD19 & B lymphocyte subpopulation & $10-15 \%$ \\
\hline CD16+CD56+ & NK - natural killer subpopulation & $13-22 \%$ \\
\hline
\end{tabular}

Table 2. Correlations between HRV parameters and antigen occurrence

\begin{tabular}{|c|c|c|c|c|c|c|}
\hline & CD3+ & CD3+ HLA-DR & CD3+ CD4+ & CD3- CD19+ & CD3+ CD8+ & CD3-CD16+ CD56+ \\
\hline $\mathbf{L F}$ & $-0.678^{*}$ & $-0.750 *$ & -0.124 & 0.157 & $-0.639^{*}$ & $0.813 * *$ \\
\hline HF & $-0.690 *$ & $-0.664 *$ & -0.411 & 0.335 & -0.530 & 0.750* \\
\hline LF/HF & 0.216 & 0.029 & $0.695^{*}$ & -0.111 & -0.188 & -0.118 \\
\hline TP & $-0.672 *$ & $-0.628 *$ & -0.343 & -0.025 & -0.533 & 0.731* \\
\hline $\mathbf{L F}(\mathbf{d})$ & -0.545 & $-0.710 *$ & 0.193 & -0.119 & $-0.805 * *$ & $0.641 *$ \\
\hline HF (d) & -0.542 & $-0.676^{*}$ & -0.191 & -0.010 & $-0.702^{*}$ & 0.466 \\
\hline LF/HF (d) & 0.264 & 0.304 & 0.583 & -0.153 & -0.008 & -0.178 \\
\hline $\mathbf{L F}(\mathbf{n})$ & $-0.702 *$ & $-0.675^{*}$ & -0.304 & 0.009 & -0.497 & $0.841 * *$ \\
\hline HF (n) & $-0.697 *$ & -0.598 & -0.472 & -0.041 & -0.425 & $0.796^{* *}$ \\
\hline LF/HF (n) & 0.200 & -0.068 & $0.690 *$ & 0.217 & -0.191 & -0.104 \\
\hline
\end{tabular}

$p<0.05^{*}$ 
Table 3. Correlation between quality of life assessment and HRV parameters

\begin{tabular}{|c|c|c|c|c|c|c|c|c|c|c|c|c|c|c|}
\hline & SDNN & SDANN & rMSSD & pNN50 & $\mathbf{L F}$ & HF & LF/HF & $\mathbf{T P}$ & LFD & HFD & LF/HFD & LFN & HFN & LFHFN \\
\hline $\begin{array}{c}\text { EQ5D - } \\
\text { pain } \cong\end{array}$ & -0.432 & -0.289 & $-0.907 * *$ & $-0.893 * *$ & 0.202 & -0.081 & 0.206 & 0.466 & 0.232 & 0.123 & 0.276 & 0.292 & -0.089 & 0.139 \\
\hline $\begin{array}{l}\text { EQ5D - } \\
\text { anxiety }\end{array}$ & -0.132 & 0.132 & -0.451 & -0.372 & 0.279 & $0.815^{*}$ & -0.346 & $0.795^{*}$ & -0.086 & 0.378 & -0.278 & $0.732 *$ & $0.838^{*}$ & -0.359 \\
\hline SWLS & $-0.812 *$ & $-0.848^{*}$ & -0.217 & -0.196 & -0.173 & -0.003 & -0.12 & $-0.730 *$ & -0.125 & 0.704 & -0.373 & -0.303 & -0.167 & -0.121 \\
\hline
\end{tabular}

deficits. Although those tests are "researcher-friendly", their psychometric value is rather poor according to the lack of the sensitivity needed to detect some subtle deficits which may stay in relation with some heart diseases [24]. It has to be mentioned that cognitive deficits in heart diseases can be related to patients' age. Very often, because of getting older, the physician observed multiple comorbid medical problems. Commonly, depression is diagnosed in heart disease. Meta-analysis of 36 studies have proved that concomitance reaches even $21.5 \%$ [25].

Patients diagnosed with heart rate disturbances performed poorer in psychological assessment involving quality of life questionnaires. Interaction between age and the disease explain difficulties in daily activities precluding selfcare behaviors as a result of executive functioning impairment. Limitation in daily activities become the main cause of decreased quality of life of cardiologic patients [26]. Additionally, low cardiac output is also related with impairment in cognitive performance and dementia [27]. Although mean values of quality of life questionnaires did not show significant differences, EQ5D parameters as perception of pain and anxiety correlated with HRV parameters. Perception of pain strongly correlated with low parasympathetic activity which may be explained by chronic tension [28]. Anxiety related to autonomic nervous system activity with predominance of sympathetic activity can also be explained by stress and physical and emotional tension, which stand alongside literature references. Emotional tension stands also beside low satisfaction with life and correlates with sympathetic activity [29, 30]. Properly chosen medical treatment of heart diseases is crucial to neuropsychological assessment outcomes. According to that fact, fluctuations in cognitive performance in heart disease patients may depend on management of the disease.

Another important aspect of heart diseases is personality trait risk factors related with cardiovascular problems. Many studies have shown that negative emotions, like anger, are correlated with cardiac diseases. Commonly used questionnaires evaluate type $\mathrm{D}$ personality which is characterized by tendency to experience negative emotions and not to express these emotions in social interactions, strongly correlates with heart diseases [31]. Other studies, concentrated on classic personality characteristic (according to the Big Five Theory), revealed that higher extraversion is correlated with an increased risk of a stroke. On the other hand, high neuroticism is associated more with the risk of a coronary heart disease than a stroke, whereas high conscientiousness is less related with a mortality risk from both coronary heart disease and stroke. A low level of agreeableness is linked to the cardiovascular diseases. Those findings lead to an assumption that personality trait links vary between main cardiac and cerebral diseases. Cardiovascular disease patients are more likely to be characterized by extraversion and openness probably according to personal predisposition to undertaking hazardous activities [32].

Psychological assessment concludes that personality traits can result in decreased quality of life and symptoms of anxiety and depression independent of traditional biomedical risk factors, including disease severity [33]. Own studies have shown that mean values of psychological assessment including personality traits are significantly different in the hypertension group and hypertension with concomitant heart rate disturbances. Health locus of control describe the hypertensive group with heart rate disturbances as more self-oriented, autonomic and responsible patients. It is due to the desire to maintain the health through health-related behaviors. It has to be mentioned that the general attitude does not always provide specific behavior [34]. Assessment of psychological comfort showed that the hypertensive group (without heart rate disturbances) manage better with daily difficulties and problems. The "heart rate disturbances group" describe their life as much more challenging and stressful. According to personality theories, the hypertensive group is characterized by the extraversion trait - it can be explained by the fact that those patients are more concentrated on positive emotions in comparison to those with heart rate disturbances. Results of psychological assessment maintain in relation to literature references, as far as all those parameters affect effectiveness of daily functioning and thereby quality of life [35].

Immunological studies have shown that heart rate disturbances cause the increase in proinflammatory cytokines. It is believed that those molecules influence the nervous system at central and peripheral levels through the activation of HPA axis. According to this fact, their probable influence on decreasing the heart rate variability index in cardiac diseases is also suggested. HPA axis affects the 
immunological status through the increased outflow of the sympathetic (SNS) and parasympathetic nervous system [36]. Researchers suggest that SNS activation exhibits a protective attribute to the organism against detrimental effects of proinflammatory cytokines. Additionally, an association between the parasympathetic nervous system and immunoregulatory processes has been suggested [37]. Many studies confirmed observation of correlation between inflammatory marker concentration and morbidity in healthy subjects and those diagnosed with cardiovascular disease [38]. First studies that confirmed relationships between HRV and biomarkers IL- 6 , TNF- $\alpha$ conducted by Aronson conceded decompensated heart failure [39]. Clinical studies conducted among arrhythmia patients also emphasize that changing levels of all biomarkers (significant increase) is correlated with such HRV parameters in time domain as standard deviation of all N-N intervals (SDNN) and metrics in the frequency domain such as LF (low frequency $0.04-0.15 \mathrm{~Hz}$ ) and $\mathrm{HF}$ (high frequency 0.15-0.4 $\mathrm{Hz}$ ). Interestingly, LF component correlation with circulating levels of TNF- $\alpha$ was higher and TNF- $\alpha$ was a significant predictor of reducing HRV value [38]. According to these results, scientists reported that overexpression of TNF- $\alpha$ and subsequent loss of $\beta$-adrenergic responsiveness lead to decreasing of HRV metrics, characteristic for cardiac diseases [40]. The increase in the percentage of CD3 cells may be indicative of excessive activity of the immune system. It occurs frequently in patients with autoimmune diseases. Negative correlations with main parameters of HRV analysis confirm dependence of CD3 on a relatively good health status. Correlations of immune parameters and HRV parameters indicate interdependence of both systems. An increase in sympathetic activity is associated with greater immune system reactivity [41].

The problem of psyche impact on different cardiovascular diseases is a subject of intensive research. Cardiovascular diseases, depressive disorder and chronic stress are among the most common health problems in highly developed countries [42]. Those illnesses are accompanied by cognitive impairment and increase in immune activation markers. Despite a significant progress in pharmacological treatment, hypertension and heart rate disturbances are still the major problem not only medical, but also psychosocial [43]. It is worth mentioning that individual differences in experiencing negative results of stressful events, including somatic afflictions as a result of cardiac disease, are not only conditioned by the general health status but also by psychological predispositions. This weakness is accompanied by cognitive functioning problems/diseases and an increase in the activity of vegetative system and immunological process markers [44, 45].

There was no complete neuropsychological assessment. The necessity of complementing knowledge with comprehensive elaboration of quality of life with consideration of psychophysiological parameters changes among cardiovas- cular patients, justifies the importance of carrying out researches concentrated on the holistic approach. Own studies evaluated psychoneuroimmunological aspects of functioning and quality of life among patients diagnosed with primary hypertension and with concomitant heart rate disturbances. The analysis of obtained results enables evaluation of not only the impact of disease symptoms, but also a proposal of further studies including proposal of effective therapy in the context of psychoneuroimmunological parameters changes. The main limitation of own studies is the sample size. It has to be mentioned that this preliminary report confirms some changes. The intended studied group contains three different samples: patients with heart rate disturbances (I), hypertension (II) and both diseases (III).

\section{Conclusions}

The pilot study has proved that:

1. Cardiovascular disease is characterized by reduced autonomic nervous system activity.

2. Impaired modulation of the autonomic nervous system activity, emerged as relative or absolute predominance of the sympathetic nervous system in a group of people diagnosed with heart rhythm disturbances affects mood, decreases quality of life and impairs attention.

3. In patients with heart rhythm disturbances, increased sympathetic nervous system activity affects prolonged tension and the immune response.

\section{The authors declare no conflict of interest.}

\section{References}

1. Kreatsoulas C, Anand SS (2010): The impact of social determinants on cardiovascular disease. Can J Cardiol 26: 8C-13C.

2. Thayer JF, Lane RD (2009): Claude Bernard and the heartbrain connection: further elaboration of a model of neurovisceral integration. Neurosci Biobehav Rev 33: 81-88.

3. Musselman DL, Evans DL, Nemeroff CB (1998): The relationship of depression to cardiovascular disease: epidemiology, biology, and treatment. Arch Gen Psychiatry 55: 580-592.

4. Mancia G, Fagard R, Narkiewicz K, et al. (2013): Wytyczne ESH/ESC dotyczące postępowania w nadciśnieniu tętniczym w 2013 roku. Arter Hypertens 17: 69-168.

5. Heras MM, Rodríguez N del C, González JF (2012): The renin-angiotensin-aldosterone system in renal and cardiovascular disease and the effects of its pharmacological blockade. J Diabetes Metab 2012.

6. Yildirir A, Batur MK, Oto A (2002): Hypertension and arrhythmia: blood pressure control and beyond. Eurpace 4: 175-182.

7. PhD NM. Cardiovascular Diseases: Nutritional and Therapeutic Interventions. CRC Press; 2013.

8. Ammirati E, Cianflone D, Vecchio V, et al. (2012): Effector Memory T cells Are Associated With Atherosclerosis in $\mathrm{Hu}-$ mans and Animal Models. J Am Heart Assoc 1: 27-41. 
9. Libby P, Ridker PM, Maseri A (2002): Inflammation and atherosclerosis. Circulation 105: 1135-1143.

10. Ribatti D, Crivellato E, Vacca A (2006): Miller's seminal studies on the role of thymus in immunity. Clin Exp Immunol 144: 371-375.

11. Dąbrowski M, Dąbrowski M, Stankiewicz W (2011): The thymus in neuro-endocrine-immune network. Centr Eur J Immunol 188-192.

12. Hadden JW (1998): Thymic endocrinology. Ann N Y Acad Sci 840: 352-358.

13. Guzik TJ, Hoch NE, Brown KA, et al. (2007): Role of the $\mathrm{T}$ cell in the genesis of angiotensin II induced hypertension and vascular dysfunction. J Exp Med 204: 2449-2460.

14. Madhur MS, Harrison DG (2013): Senescent T cells and hypertension: new ideas about old cells. Hypertension 62: 13-15.

15. Deschaux P, Rouabhia M (1987): The thymus. Key organ between endocrinologic and immunologic systems. Ann N Y Acad Sci 496: 49-55.

16. Asadi-Lari M, Tamburini M, Gray D (2004): Patients' needs, satisfaction, and health related quality of life: Towards a comprehensive model. Health Qual Life Outcomes 2: 32.

17. Roditi D, Robinson ME (2011): The role of psychological interventions in the management of patients with chronic pain. Psychol Res Behav Manag 4: 41-49.

18. Hlatky MA, Vaughn WK (1996): Quality of life in patients with supraventricular arrhythmia. Circulation 94: 1491-1493.

19. AbuRuz ME, Alaloul F, Saifan A, et al. (2015): Quality of life for saudi patients with heart failure: a cross-sectional correlational study. Glob J Health Sci 8: 45752.

20. Gillie BL, Thayer JF (2014): Individual differences in resting heart rate variability and cognitive control in posttraumatic stress disorder. Front Psychol 5: 758.

21. McCorry LK (2007): Physiology of the autonomic nervous system. Am J Pharm Educ 2007; 71.

22. Hovland A, Pallesen S, Hammar Ĺ, et al. (2012): The relationships among heart rate variability, executive functions, and clinical variables in patients with panic disorder. Int J Psychophysiol 86: 269-275.

23. Kim DH, Lipsitz LA, Ferrucci L, et al. (2006): Association between reduced heart rate variability and cognitive impairment in older disabled women in the community: women's health and aging study I. J Am Geriatr Soc 54: 1751-1757.

24. Garin O, Ferrer M, Pont Ŕ, et al. (2008): Disease-specific health-related quality of life questionnaires for heart failure: a systematic review with meta-analyses. Qual Life Res 18: 71-85.

25. Ski CF, Jelinek M, Jackson AC, et al. (2015): Psychosocial interventions for patients with coronary heart disease and depression: A systematic review and meta-analysis. Eur J Cardiovasc Nurs doi:10.1177/1474515115613204.

26. Szyguła-Jurkiewicz B, Kowalska M, Mościński M (2011): Jakość życia jako element oceny stanu zdrowia i efektywności leczenia chorych ze schorzeniami układu sercowo-naczyniowego. Folia Cardiol 6: 62-71.

27. Dardiotis E, Giamouzis G, Mastrogiannis D, et al. (2012): Cognitive impairment in heart failure, cognitive impairment in heart failure. Cardiol Res Pract 2012: e595821.

28. Tracy LM, Ioannou L, Baker KS, et al. (2016): Gibson SJ, Georgiou-Karistianis N, Giummarra MJ. Meta-analytic evidence for decreased heart rate variability in chronic pain implicating parasympathetic nervous system dysregulation. Pain 157: 7-29.
29. Chalmers JA, Quintana DS, Abbott MJ-A, Kemp AH (2014): Anxiety disorders are associated with reduced heart rate variability: a meta-analysis. Affect Disord Psychosom Res 5: 80.

30. Kreibig SD (2010): Autonomic nervous system activity in emotion: A review. Biol Psychol 84: 394-421.

31. Kupper N, Pedersen SS, Höfer S, et al. (2013): Cross-cultural analysis of type D (distressed) personality in 6222 patients with ischemic heart disease: a study from the International HeartQoL Project. Int J Cardiol 166: 327-333.

32. Jokela M, Pulkki-Ríback L, Elovainio M, Kivimäki M (2014): Personality traits as risk factors for stroke and coronary heart disease mortality: pooled analysis of three cohort studies. J Behav Med 37: 881-889.

33. Khayyam-Nekouei Z, Neshatdoost H, Yousefy A, et al. (2013): Psychological factors and coronary heart disease. ARYA Atheroscler 9: 102-111.

34. Omeje O, Nebo C (2011): The influence of locus control on adherence to treatment regimen among hypertensive patients. Patient Prefer Adherence 5: 141-148.

35. Hawthorne MH, Hixon ME (1993): Functional status, mood disturbance and quality of life in patients with heart failure. Prog Cardiovasc Nurs 9: 22-32.

36. Straburzyńska-Migaj E, Ochotny R, Wachowiak-Baszyńska A, et al. (2005): Cytokines and heart rate variability in patients with chronic heart failure. Kardiol Pol 63: 478-485.

37. Kenney MJ, Ganta CK (2014): Autonomic nervous system and immune system interactions. Compr Physiol 2014; 4: 1177-1200.

38. Papaioannou V, Pneumatikos I, Maglaveras N (2013): Association of heart rate variability and inflammatory response in patients with cardiovascular diseases: current strengths and limitations. Front Physiol 2013; 4: 174.

39. Janszky I, Ericson M, Lekander M, et al. (2004): Inflammatory markers and heart rate variability in women with coronary heart disease. J Intern Med 2004; 256: 421-428.

40. Papaioannou VE, Verkerk AO, Amin AS, de Bakker JM (2013): Intracardiac origin of heart rate variability, pacemaker funny current and their possible association with critical illness. Curr Cardiol Rev 9: 82-96.

41. Norman RA, Dzielak DJ (1986): Immunological dysfunction and enhanced sympathetic activity contribute to the pathogenesis of spontaneous hypertension. J Hypertens Suppl 4: S437-S439.

42. Albus C, Ladwig K-H, Herrmann-Lingen C (2014): Psychocardiology: clinically relevant recommendations regarding selected cardiovascular diseases. Dtsch Med Wochenschr 1946 139: 596-601.

43. Cuffee Y, Ogedegbe C, Williams NJ, et al. (2014): Psychosocial risk factors for hypertension: an update of the literature. Curr Hypertens Rep 16: 483.

44. Gouin JP, Wenzel K, Boucetta S, et al. (2015): High-frequency heart rate variability during worry predicts stress-related increases in sleep disturbances. Sleep Med 16: 659-664.

45. Moryś JM, Bellwon J, Jeżewska M, et al. (2015): The evaluation of stress coping styles and type D personality in patients with coronary artery disease. Kardiol Pol 73: 557-566. 